\title{
Reutilização do Acesso Transradial na Realização de Procedimentos Coronários Diagnósticos
}

\author{
Marden Andre Tebet ${ }^{1,2}$, Pedro Beraldo de Andrade ${ }^{1,2}$, Monica Vieira Athanazio de Andrade ${ }^{1}$, \\ Luiz Alberto Mattos ${ }^{1,3}$, André Labrunie ${ }^{1,2}$
}

\section{RESUMO}

Introdução: $O$ acesso transradial tem obtido maior aceitação e interesse pela menor ocorrência de sangramentos e de complicações vasculares locais e pelo maior conforto ao paciente. Em centros que o utilizam como sua via preferencial, muitos pacientes necessitarão do reuso dessa via. Esta análise tem como objetivo avaliar a segurança, a eficácia e as limitações de sua reutilização na prática diária. Método: Foram avaliados pacientes consecutivos submetidos a procedimentos coronários diagnósticos por acesso radial, incluídos em um registro prospectivo. Comparamos pacientes que utilizaram a via radial por mais de uma vez (grupo I) com os que a utilizaram de maneira única (grupo II), avaliando o sucesso da técnica, a duração do procedimento e da fluoroscopia, as complicações vasculares e os eventos cardiovasculares adversos graves. Adicionalmente, obtivemos no grupo I a frequência, o sucesso e as limitações no número de repetições. Resultados: Entre maio de 2008 e dezembro de 2009, 232 pacientes com utilização prévia do acesso radial direito foram submetidos a coronariografia pela mesma via e comparados a 1.857 pacientes com utilização única. A taxa de sucesso do procedimento $(99,5 \%$ vs. $98,1 \% ; P=0,17)$ e a duração do exame $(15,78 \pm 6,8 \mathrm{~min}$ vs. $15,54 \pm 7,7 \mathrm{~min}$; $\mathrm{P}=0,62)$ e da fluoroscopia $(3,26 \pm 3,01 \mathrm{~min}$ vs. $3,26 \pm$ $2,42$ min; $P>0,99)$ foram semelhantes entre os grupos. $O$ sucesso do procedimento também não foi diferente entre os pacientes do grupo I com $\geq 2$ reutilizações, quando comparados àqueles com única reutilização $(99,2 \%$ vs. $100 \% ; P=0,99)$. Na análise tardia do grupo I, 602 procedimentos foram realizados com taxa de sucesso de $98,9 \%$, embora com diminuição não-significativa da taxa de sucesso a cada repetição, até a quarta reutilização. Conclusão: A reutilização do acesso radial na realização de procedimentos coronários diagnósticos mostra-se efetiva, considerando-se sua alta taxa de sucesso e a baixa

\begin{abstract}
Repeat Transradial Access in Diagnostic Coronary Procedures

Background: Transradial access has gained greater acceptance and interest due to the lower incidence of bleeding and local vascular complications and greater comfort for the patient. In centers where it is used as the preferred access, repeat transradial procedures are becoming routine. This study is aimed at assessing the safety, efficacy and limitations of repeated procedures in the daily practice. Method: Consecutive patients undergoing diagnostic coronary procedures using the transradial access were included in a prospective registry. Those who used the radial access more than once (group I) were compared with those who used it at one time only (group II). The success of the technique, procedural and fluoroscopic times, vascular complications and major adverse cardiovascular events were evaluated. In addition, in group I we obtained the frequency, success and limitations in the number of repeated procedures. Results: From May 2008 to December 2009, 232 patients with previous right transradial access underwent diagnostic coronary procedures using the same access route and were compared to 1,857 patients who used it at one time only. The procedure success rate $(99.5 \%$ vs. $98.1 \% ; P=0.17)$, the procedural time $(15.78 \pm 6.8 \mathrm{~min}$ vs. $15.54 \pm 7.7 \mathrm{~min} ; \mathrm{P}=0.62)$ and fluoroscopic time $(3.26 \pm 3.01 \mathrm{~min}$ vs. $3.26 \pm 2.42 \mathrm{~min}$; $\mathrm{P}>0.99$ ) were similar between groups. The procedure success rate did not differ among group I patients with $\geq 2$ repetitions, when compared with those who used it at one time only $(99.2 \%$ vs. $100 \% ; \mathrm{P}=0.99)$. In the late follow-up of group I patients, 602 procedures were carried out with a success rate of $98.9 \%$, although there was a non-significant decrease of the success rate after each repetition up to the fourth procedure. Conclusion: The reuse of transradial
\end{abstract}

1 Irmandade da Santa Casa de Misericórdia de Marília - Marília, SP, Brasil.

2 Hospital do Coração de Londrina - Londrina, PR, Brasil.

3 Instituto Dante Pazzanese de Cardiologia - São Paulo, SP, Brasil. Correspondência: Marden A. Tebet. Irmandade da Santa Casa de Misericórdia de Marília. Avenida Vicente Ferreira, 828 - Bairro Cascata - Marília, SP, Brasil - CEP 17515-900

E-mail: mardentebet@gmail.com

Recebido em: 17/12/2009 • Aceito em: 22/2/2010 
taxa de complicações na maioria dos pacientes, embora com limitação do número de reutilizações a cada procedimento adicional.

DESCRITORES: Artéria radial. Angiografia coronária.

A pós sua introdução para realização de procedimentos coronários diagnósticos, em 1989, por Campeau, ${ }^{1}$ e terapêuticos, em 1993, por Kiemeneij e Laarman, ${ }^{2}$ o acesso radial estabeleceu-se como via segura e custo-efetiva quando comparado às técnicas femoral e braquial. Embora tecnicamente mais desafiador, como demonstrado por sua curva de aprendizagem, ${ }^{3,4}$ associa-se a significativa redução das taxas de sangramento e complicações relacionadas ao sítio de punção. ${ }^{5,6}$ Propicia maior conforto ao paciente no período de recuperação, além de deambulação e alta precoces, com consequente redução dos custos hospitalares. ${ }^{7}$

Entretanto, embora infrequentes, podem ocorrer complicações após a utilização do acesso radial. A incidência de oclusão da artéria radial é de 5,3\% agudamente e de $2,8 \%$ cronicamente. ${ }^{8}$ Ademais, muitos pacientes nos quais a via radial é utilizada apresentam doença vascular em outros sítios e necessitarão de procedimentos vasculares percutâneos repetidos durante toda sua vida, sendo importante a avaliação da reutilização do acesso radial nos serviços que o utilizam como sua via principal.

O objetivo do estudo é avaliar a reutilização do acesso radial na prática diária, sua frequência e limitações, comparativamente a sua utilização única.

\section{MÉTODO}

Pacientes consecutivos submetidos a procedimentos cardíacos diagnósticos e terapêuticos foram incluídos em um registro prospectivo. Nos pacientes que utilizaram a via radial, avaliamos aqueles submetidos a cineangiocoronariografias, comparando os que reutilizaram o mesmo acesso mais de uma vez com aqueles que o utilizaram em um único procedimento. Pacientes submetidos a procedimentos adicionais (estudo de pontes, cateterismo direito, arteriografia de carótidas e aortografia) foram excluídos da presente análise.

A eficácia da técnica radial foi avaliada por meio da taxa de sucesso do procedimento, definida como realização de cinecoronariografia com adequada opacificação coronária sem necessidade de mudança da via de acesso (crossover). Os tempos de procedimento e fluoroscopia foram obtidos a partir do início da punção arterial até a retirada do introdutor. A segurança foi avaliada por meio da ocorrência de complicações vasculares relacionadas ao sítio de punção e access in diagnostic coronary procedures has proven to be effective, taking into consideration its high success rate and low complication rates in most of the patients, despite the limitation in the number of repetitions after each additional procedure.

KEY-WORDS: Radial artery. Coronary angiography.

eventos cardiovasculares adversos graves periprocedimento. Por fim, obtivemos no seguimento tardio análise do intervalo entre os procedimentos, número de procedimentos realizados pelo mesmo acesso e seus resultados.

Por meio de hiperextensão do punho e infiltração de $1 \mathrm{ml}$ a $2 \mathrm{ml}$ de xilocaína a $2 \%$, puncionava-se a artéria radial $1 \mathrm{~cm}$ proximal ao processo estiloide do rádio, utilizando-se agulha com cateter de polietileno tipo Jelco ${ }^{\mathrm{TM}}$ calibre 20 e técnica de Seldinger. Após a punção, introduzia-se um fio-guia de 0,021 polegada, seguido de pequena incisão cutânea com lâmina de bisturi $\mathrm{n}^{\mathrm{o}} 11$ e inserção de introdutor curto $(<11 \mathrm{~cm})$ 5-7 F. Administrava-se uma solução contendo 5.000 UI de sulfato de heparina e $10 \mathrm{mg}$ de mononitrato de isossorbida através da extensão do introdutor. Ao término do procedimento, removia-se imediatamente o introdutor e obtinha-se hemostasia com curativo compressivo por meio de bandagem elástica adesiva porosa (Tensoplast ${ }^{\mathrm{TM}}$ ). Procedia-se a exame clínico do sítio de punção e avaliação do pulso radial no momento da alta, cerca de duas a três horas após a realização de procedimentos diagnósticos.

Classificou-se como evento cardiovascular adverso grave a ocorrência de morte, infarto agudo do miocárdio, acidente vascular encefálico, cirurgia de revascularização miocárdica de emergência ou sangramento grave. Este foi definido como hemorragia intracraniana, intraocular, retroperitoneal, queda de hemoglobina superior a $3 \mathrm{~g} / \mathrm{dl}$ ou necessidade de transfusão sanguínea. Os hematomas foram graduados de acordo com a classificação do estudo Early Discharge after Transradial Stenting of Coronary Arteries (EASY) ${ }^{9}$ : tipo I, $<5 \mathrm{~cm}$ de diâmetro; tipo II, $<10 \mathrm{~cm}$ de diâmetro; tipo III, $>10 \mathrm{~cm}$, sem atingir o cotovelo; tipo IV, hematoma estendendo-se além do cotovelo; e tipo $\mathrm{V}$, qualquer hematoma com injúria isquêmica à mão. Complicações relacionadas ao sítio de punção além de hematomas incluíram fístula arteriovenosa, pseudoaneurisma, oclusão arterial assintomática, necessidade de reparo vascular cirúrgico e infecção local.

As variáveis contínuas foram expressas em médias e desvio padrão e comparadas com o test $t$ de Student. As variáveis categóricas foram expressas em frequência e porcentagem, comparadas com o teste de qui-quadrado ou o teste exato de Fisher, quando apropriado. Considerou-se estatisticamente significante $\mathrm{P}<0,05$. 
Tebet MA, et al. Reutilização do Acesso Transradial na Realização de Procedimentos Coronários Diagnósticos. Rev Bras Cardiol Invasiva. 2010;18(1):37-43.

\section{RESULTADOS}

No período de maio de 2008 a dezembro de 2009, 3.255 pacientes foram submetidos a procedimentos cardíacos invasivos diagnósticos e terapêuticos em nosso serviço, sendo o acesso radial utilizado em $91,8 \%$ deles. Nos submetidos a cineangiocoronariografia, comparamos os que reutilizaram o mesmo acesso mais de uma vez (grupo I, $\mathrm{n}=232$ ) com aqueles que o utilizaram em um único procedimento (grupo $\mathrm{II}, \mathrm{n}=1.857$ ).

As características demográficas, clínicas e do procedimento dos pacientes que realizaram coronariografia estão expressas na Tabela 1. Houve predomínio de pacientes com hipertensão arterial (87,9\% vs. 79\%; $P=0,002)$, diabetes melito $(37,9 \%$ vs. $26,6 \%$; $P<0,001)$, dislipidemia $(63,3 \%$ vs. 42,2\%; $\mathrm{P}<0,001)$, infarto agudo do miocárdio prévio (28,8\% vs. 7,5\%; $\mathrm{P}<0,001)$, intervenção coronária percutânea prévia $(53,4 \%$ vs. $2,5 \% ; P<0,001)$ e cateterismo prévio pela técnica de Sones $(24,1 \%$ vs. 9,7\%; P $<0,001)$ no grupo I. Por outro lado, houve maior frequência de mulheres $(37 \%$ vs. $44,5 \%$; $P=0,04)$ e de tabagismo $(18,5 \%$ vs. $26,3 \%$; $\mathrm{P}=0,012)$ no grupo II. Não houve diferenças estatisticamente significantes quanto a idade, peso, altura e apresentação clínica dos pacientes.

A taxa de sucesso em ambos os grupos foi similar (99,5\% no grupo I e 98,1\% no grupo $\mathrm{II}$; $\mathrm{P}=0,17)$, assim como não houve diferenças nas demais características do procedimento (duração do exame e da fluoroscopia, uso de sedativos, e número e diâmetro dos cateteres). Ocorreu um insucesso no grupo I por não progressão do cateter, sendo o exame realizado pela via ulnar esquerda. As causas mais frequentes de insucesso no grupo II foram a presença de oclusão pós-arteriotomia braquial prévia (27\%), espasmo radial (25\%), hipoplasia e oclusão de artéria radial (17\%), oclusão de tronco braquiocefálico ou subclávia (14\%) e trajeto anômalo da artéria radial (11\%). O acesso radial esquerdo foi a via alternativa escolhida em 55\% dos casos em que foi necessária a mudança da via de acesso.

TABELA 1

Características demográficas, clínicas e do procedimento

\begin{tabular}{|c|c|c|c|}
\hline Variáveis & $\begin{array}{c}\text { Grupo I } \\
(n=232)\end{array}$ & $\begin{array}{c}\text { Grupo II } \\
(n=1.857)\end{array}$ & $\mathbf{P}$ \\
\hline Idade, anos & $61,5 \pm 10,8$ & $60,71 \pm 11,1$ & 0,31 \\
\hline Sexo feminino, n (\%) & $86(37)$ & $826(44,5)$ & 0,04 \\
\hline Peso, kg & $75,85 \pm 15,95$ & $74,65 \pm 15,54$ & 0,27 \\
\hline Altura, m & $1,65 \pm 0,08$ & $1,64 \pm 0,08$ & 0,07 \\
\hline Hipertensão arterial sistêmica, n (\%) & $204(87,9)$ & $1.468(79)$ & 0,002 \\
\hline Diabetes melito, n (\%) & $88(37,9)$ & $495(26,6)$ & $<0,001$ \\
\hline Dislipidemia, n (\%) & $147(63,3)$ & $785(42,2)$ & $<0,001$ \\
\hline Tabagismo, n (\%) & $43(18,5)$ & $489(26,3)$ & 0,012 \\
\hline Histórico familiar de insuficiência coronária, n (\%) & $49(21,1)$ & $346(18,6)$ & 0,41 \\
\hline Infarto agudo do miocárdio prévio, n (\%) & $67(28,8)$ & $139(7,5)$ & $<0,001$ \\
\hline Cateterismo prévio (técnica de Sones - MSD), n (\%) & $56(24,1)$ & $180(9,7)$ & $<0,001$ \\
\hline Intervenção coronária percutânea prévia, n (\%) & $124(53,4)$ & $46(2,5)$ & $<0,001$ \\
\hline Acidente vascular encefálico prévio, n (\%) & $7(3,1)$ & $55(2,9)$ & 0,96 \\
\hline Quadro clínico, n (\%) & & & 0,36 \\
\hline Angina estável/isquemia silenciosa & $125(53,8)$ & $845(45,5)$ & \\
\hline SCA sem supradesnível de ST & $72(31)$ & $586(31,5)$ & \\
\hline SCA com supradesnível de ST & $7(3,1)$ & $35(1,9)$ & \\
\hline Sucesso do procedimento, n (\%) & $231(99,5)$ & $1,821(98,1)$ & 0,17 \\
\hline Duração do procedimento, min & $15,78 \pm 6,8$ & $15,54 \pm 7,7$ & 0,62 \\
\hline Duração da fluoroscopia, min & $3,26 \pm 3,01$ & $3,26 \pm 2,42$ & $>0,99$ \\
\hline Número de cateteres utilizados, média \pm DP & $2 \pm 0,53$ & $2,01 \pm 0,54$ & 0,79 \\
\hline Utilização de sedação, n (\%) & $22(9,5)$ & $136(7,3)$ & 0,30 \\
\hline Diâmetro do cateter, \% & & & 0,33 \\
\hline $5 \mathrm{~F}$ & 86,2 & 88,5 & \\
\hline $6 \mathrm{~F}$ & 13,8 & 11,5 & \\
\hline
\end{tabular}

$\mathrm{DP}=$ desvio padrão; $\min =$ minutos; $\mathrm{MSD}=$ membro superior direito; $\mathrm{n}=$ número de pacientes; $\mathrm{SCA}$ = síndrome coronária aguda . 
Dentre as complicações observadas com a utilização do acesso radial (Tabela 2) a mais comum foi a oclusão arterial assintomática, sem repercussão isquêmica da mão $(2,1 \%$ no grupo I e $1,67 \%$ no grupo II; $\mathrm{P}=0,79)$. Espasmo grave ocorreu em $1,3 \%$ no grupo I e em $1,4 \%$ no grupo II $(P=0,85)$ e hematomas ocorreram em $2,1 \%$ no grupo I e em $1,77 \%$ no grupo II $(P=0,58)$, sendo a maioria do tipo I, restritos ao tecido subcutâneo e inferiores a $5 \mathrm{~cm}$ de extensão. Houve um $(0,1 \%)$ episódio de dissecção de tronco de coronária esquerda durante coronariografia, evoluindo com infarto do miocárdio, com necessidade de cirurgia cardíaca de emergência e que resultou em óbito. Não ocorreram em quaisquer dos grupos pseudoaneurismas, fístulas arteriovenosas, sangramentos graves ou transfusões sanguíneas.

O intervalo médio entre o procedimento inicial e a reutilização do acesso, no grupo I, em pacientes submetidos a coronariografia foi de $8,7 \pm 23$ dias. A distribuição do intervalo foi de 1 dia em 53,4\%, de $2-3$ dias em 18,4\%, de 4-10 dias em 14,5\%, de 10-30 dias em $6,8 \%$, e de mais de 30 dias em $6,8 \%$.
Em análise adicional dos pacientes que reutilizaram o acesso radial (Tabela 3), encontramos insucesso do procedimento em único paciente que reutilizou esse acesso somente uma vez. Não encontramos diferenças nas características do procedimento entre aqueles que reutilizaram a via radial uma única vez ou mais de uma vez.

Após o último procedimento, no grupo I, continuamos o seguimento de cada um desses pacientes até seu último procedimento no registro para avaliar se houve algum insucesso posterior. Da amostra total de 232 pacientes, 10 estavam presentes de maneira duplicada por procedimentos em dois momentos do registro, tendo sido excluído o primeiro exame desses pacientes. Nesse grupo de pacientes, houve um total de 602 procedimentos, distribuídos conforme demonstrado na Tabela 4. A maioria dos pacientes $(85,5 \%)$ não realizou procedimento adicional no seguimento do registro, nos quais a taxa de sucesso foi de $98,9 \%$. Naqueles que o fizeram, a taxa de sucesso foi de $84,4 \%$ $(\mathrm{P}<0,001)$. Dos pacientes que reutilizaram o acesso radial uma única vez (2 procedimentos), 111 (91\%) não

TABELA 2

Taxa de complicações periprocedimento com a utilização do acesso radial

\begin{tabular}{lccc}
\hline Variáveis & $\begin{array}{c}\text { Grupo I } \\
(\mathbf{n = 2 3 2 )}\end{array}$ & $\begin{array}{c}\text { Grupo II } \\
(\mathbf{n}=\mathbf{1 . 8 5 7})\end{array}$ & $\mathbf{P}$ \\
\hline Espasmo grave, n (\%) & $3(1,3)$ & $27(1,4)$ & 0,85 \\
Oclusão arterial assintomática, n (\%) & $5(2,1)$ & $31(1,67)$ & 0,79 \\
Hematoma, n (\%) & & & 0,58 \\
$\quad$ Tipo I & $4(1,7)$ & $12(0,64)$ & $1(0,05)$ \\
$\quad$ Tipo II & $1(0,43)$ & $1(0,05)$ & 0,53 \\
$\quad$ Tipo III & 0 & $1(0,05)$ & 0,72 \\
Cirurgia cardíaca de emergência, n & $1(0,43)$ & 0 & $\mathrm{NA}$ \\
Infarto agudo do miocárdio, n & 0 & $1(0,05)$ & 0,73 \\
Acidente vascular encefálico/AIT, n & 0 & 0 & \\
Morte, $\mathrm{n}$ (\%) & &
\end{tabular}

AIT = ataque isquêmico transitório, $\mathrm{n}$ = número de pacientes, NA = não se aplica.

TABELA 3

Comparação das características do procedimento, de acordo com o número de reutilizações do acesso radial, em pacientes submetidos a coronariografia no grupo I

\begin{tabular}{lccc}
$\begin{array}{l}\text { Número de reutilizações } \\
(\mathbf{n}=\mathbf{2 3 2})\end{array}$ & $\begin{array}{c}\mathbf{1} \\
(\mathbf{n}=\mathbf{1 2 7})\end{array}$ & $\begin{array}{c}>\mathbf{1} \\
(\mathbf{n}=\mathbf{1 0 5})\end{array}$ & $\mathbf{P}$ \\
\hline Sucesso do procedimento, n (\%) & $126(99,2)$ & $105(100)$ & 0,99 \\
Duração do procedimento, min & $16,21 \pm 8,17$ & $15,25 \pm 4,62$ & 0,26 \\
Duração de fluoroscopia, min & $3,41 \pm 3,42$ & $3,09 \pm 1,53$ & 0,35 \\
Número de cateteres utilizados, média \pm DP & $2,01 \pm 0,60$ & $1,99 \pm 0,43$ & 0,77 \\
\hline
\end{tabular}

$\mathrm{DP}=$ desvio padrão; $\min =$ minutos; $\mathrm{n}=$ número de pacientes. 
Tebet MA, et al. Reutilização do Acesso Transradial na Realização de Procedimentos Coronários Diagnósticos. Rev Bras Cardiol Invasiva. 2010;18(1):37-43.

realizaram procedimentos adicionais e $11(9,1 \%)$ realizaram mais um. As taxas de insucesso foram de 0,45\% e $9 \%$, respectivamente $(P<0,001)$. Naqueles que reutilizaram 2 vezes (3 procedimentos), 65 (79,3\%) não realizaram novos procedimentos e 17 (20,7\%) realizaram um procedimento adicional. A taxa de insucesso foi de $0,5 \%$ e $2,9 \%$, respectivamente $(P=0,107)$. Quando a via radial foi reutilizada 3 vezes (4 procedimentos), 9 (75\%) não realizaram novo procedimento e 3 (25\%) tiveram outro procedimento. Adicionalmente, no grupo de 4 reusos (5 procedimentos),
$3(75 \%)$ não realizaram novo procedimento e 1 (25\%) realizou um procedimento a mais. No último grupo $(5$ reutilizações, 6 procedimentos), não houve procedimento adicional em dois pacientes. Não houve insucesso nos pacientes com 3, 4 e 5 reusos. Houve um total de 7 insucessos em 602 procedimentos (1,1\%). A via radial esquerda foi utilizada em 4 pacientes, e os outros procedimentos foram realizados pelas vias ulnar direita, ulnar esquerda e femoral direita. Não houve diferenças nas características basais e do procedimento nos pacientes com e sem sucesso (Tabela 5).

TABELA 4

Número total de procedimentos repetidos em pacientes submetidos a coronariografia no grupo I

\begin{tabular}{|c|c|c|}
\hline $\begin{array}{l}\text { Número total de procedimentos repetidos } \\
\text { por paciente - evolução tardia - grupo I } \\
(n=222 / p=602)\end{array}$ & $\begin{array}{l}\text { Sucesso por } \\
\text { paciente } \\
\text { n }(\%)\end{array}$ & $\begin{array}{l}\text { Sucesso por } \\
\text { procedimento } \\
\qquad \text { p (\%) }\end{array}$ \\
\hline 2 procedimentos repetidos $(n=111 / p=222)$ & $110(99,1)$ & $221(99,5)$ \\
\hline 3 procedimentos repetidos $(n=76 / p=228)$ & $72(94,7)$ & $224(98,2)$ \\
\hline 4 procedimentos repetidos $(n=26 / p=104)$ & $24(92,3)$ & $102(98,1)$ \\
\hline 5 procedimentos repetidos $(n=6 / p=30)$ & $6(100)$ & $30(100)$ \\
\hline 6 procedimentos repetidos $(n=3 / p=18)$ & $3(100)$ & $18(100)$ \\
\hline
\end{tabular}

TABELA 5

Comparação das características demográficas, clínicas e do procedimento em pacientes com e sem sucesso do procedimento submetidos a coronariografia no grupo I

\begin{tabular}{|c|c|c|c|}
\hline Variáveis & $\begin{array}{c}\text { Insucesso } \\
\mathbf{n}=7\end{array}$ & $\begin{array}{l}\text { Sucesso } \\
n=215\end{array}$ & $\mathbf{P}$ \\
\hline Idade, anos & $56,4 \pm 9,62$ & $61,74 \pm 10,79$ & 0,20 \\
\hline Sexo feminino, n (\%) & $3(42,8)$ & $77(35,8)$ & 0,70 \\
\hline Peso, kg & $69,71 \pm 19,87$ & $76,28 \pm 16,11$ & 0,29 \\
\hline Altura, m & $1,63 \pm 0,10$ & $1,65 \pm 0,08$ & 0,52 \\
\hline Hipertensão arterial sistêmica, n (\%) & $7(100)$ & $189(87,9)$ & $>0,99$ \\
\hline Diabetes melito, n (\%) & $2(28,6)$ & $80(37,2)$ & $>0,99$ \\
\hline Dislipidemia, n (\%) & $5(71,4)$ & $136(63,2)$ & $>0,99$ \\
\hline Tabagismo, n (\%) & $3(42,8)$ & $40(18,6)$ & 0,13 \\
\hline Histórico familiar de insuficiência coronária, n (\%) & 0 & $45(20,9)$ & 0,35 \\
\hline Infarto agudo do miocárdio prévio, n (\%) & $4(57,1)$ & $59(27,4)$ & 0,10 \\
\hline Cateterismo prévio (técnica de Sones - MSD), n (\%) & 0 & $55(25,5)$ & 0,20 \\
\hline Intervenção coronária percutânea prévia, n (\%) & $3(42,8)$ & $115(53,4)$ & 0,71 \\
\hline Duração do procedimento, min & $20 \pm 17,80$ & $15,76 \pm 6,31$ & 0,55 \\
\hline Duração de fluoroscopia, min & $4,49 \pm 6,59$ & $3,26 \pm 2,53$ & 0,64 \\
\hline Número de cateteres utilizados, média \pm DP & $2,43 \pm 1,13$ & $1,99 \pm 0,51$ & 0,34 \\
\hline Diâmetro do cateter, \% & & & 0,60 \\
\hline $5 \mathrm{~F}$ & 100 & 85,5 & \\
\hline $6 / 7 \mathrm{~F}$ & 0 & 14,5 & \\
\hline
\end{tabular}

$\mathrm{DP}=$ desvio padrão; $\min =$ minutos; $\mathrm{MSD}=$ membro superior direito; $\mathrm{n}$ = número de pacientes. 
Tebet MA, et al. Reutilização do Acesso Transradial na Realização de Procedimentos Coronários Diagnósticos. Rev Bras Cardiol Invasiva. 2010;18(1):37-43.

\section{DISCUSSÃO}

Os dados do registro demonstram que em um serviço cujos operadores têm extenso treinamento e realizam alto volume de procedimentos pela via radial, ${ }^{10}$ sua reutilização ocorre em cerca de $20 \%$ dos casos e pode ser realizada com a mesma eficácia e segurança da primeira vez.

A obtenção da via de acesso e a conclusão do procedimento com sucesso em ambos os grupos foram elevadas (> 98\%), sem incrementos na duração total do procedimento ou de fluoroscopia, este último um fiel marcador de dificuldade e complexidade técnica. Resultados observados mesmo nos pacientes do grupo I, em relação ao número de reutilizações, ao sucesso ou ao insucesso do procedimento. Além da eficácia, avaliou-se a segurança de nova punção da via radial, com pequeno número de complicações vasculares e nenhuma hemorragia grave no local da punção, sem diferença entre os grupos avaliados. Embora os pacientes no grupo I apresentassem maior número de fatores de risco, que poderia representar uma doença cardiovascular mais grave, não observamos aumento das taxas de insucesso e complicações nesse grupo.

Após o sucesso do primeiro procedimento, o fator mais importante no sucesso do novo procedimento é a punção arterial. A maioria dos pacientes reutilizou essa via de uma maneira precoce e grande parte desses o fez no dia seguinte ao procedimento prévio. A nova punção foi realizada tecnicamente da mesma maneira e geralmente no exato local ou o mais próximo possível da incisão do primeiro acesso, que ainda não se encontra cicatrizada. Não observamos aumento de espasmo e principalmente de complicações vasculares com essa abordagem. Em casos de maior número de repetição ou de novas punções tardias, a fibrose local poderá torná-la um pouco mais dificultosa, principalmente no momento da retração do Jelco após a transfixação da artéria radial e a retirada da agulha. Evitamos punções muito proximais ao local da primeira, pois, ao nos afastarmos do processo estiloide, essa punção se tornará mais profunda e possivelmente poderá levar ao aumento do número de complicações como hematomas e pseudoaneurismas de artéria radial.

Caputo et al. ${ }^{11}$ avaliaram 73 pacientes que reutilizaram o acesso radial em um total de 1.362 procedimentos diagnósticos. Características basais, taxas de sucesso do procedimento $(100 \%$ vs. $97,9 \% ; \mathrm{P}=\mathrm{NS})$ e tempo de procedimento $(23,9 \pm 27,3$ min vs. $18,2 \pm$ 14,7 min; $P=N S$ ) foram similares entre os grupos. Entretanto, somente os pacientes com teste de Allen normal e pulso amplo foram selecionados para a inclusão nessa avaliação.

Em nossa série, não encontramos diminuição das taxas de sucesso a cada nova punção, nem da duração do exame e da fluoroscopia ou do número de cateteres utilizados, comparativamente àqueles que reutilizaram somente uma vez. Entretanto, um potencial viés poderia ser representado pela avaliação única do número de reutilizações, conforme já descrito, pois, após o procedimento índice, o paciente poderia ter realizado outro exame por acessos que não o radial direito e não seria incluído nessa amostra. Na avaliação por procedimentos posteriores dentro do registro, encontramos diminuição do sucesso até a quarta punção, embora não estatisticamente significante, e com índice de sucesso ainda $>90 \%$. Não houve insucessos nos poucos pacientes que necessitaram realizar o exame por cinco ou seis vezes no mesmo acesso. Foi possível reutilizar o mesmo acesso em até quatro vezes (em alguns casos, em até seis vezes) em mais de $90 \%$ dos pacientes. Sakai et al. ${ }^{12}$ avaliaram 198 homens e 89 mulheres que utilizaram o acesso radial por pelo menos duas vezes e encontraram taxa de falência maior nas mulheres (7,9\% vs. 3,5\%), além de aumento gradual da taxa de falência a partir do segundo procedimento (7,9\% para $28,6 \%$ no quinto procedimento, no sexo feminino, e $3,5 \%$ para $15,6 \%$ no quinto procedimento, no sexo masculino). Foi possível a utilização de um terceiro procedimento pelo mesmo acesso em 90\% dos homens e em $80 \%$ das mulheres e a utilização de um quinto procedimento foi possível em $70 \%$ dos homens e em 50\% das mulheres.

A principal causa de falência (90\%) no estudo de Sakai et al. ${ }^{12}$ foi o estreitamento ou a oclusão da artéria radial depois do exame prévio. Isso é explicado possivelmente pelo espessamento da túnica intimal decorrente de injúria local após inserção do introdutor, resultando em estreitamento progressivo com o uso repetido do acesso radial. Yoo et al. ${ }^{13}$ avaliaram as mudanças no diâmetro da artéria radial, os resultados do procedimento e as complicações do uso repetido da via radial em 117 pacientes. Nenhuma diferença significante foi encontrada no diâmetro médio da artéria radial entre o período anterior ao procedimento e um dia após. Entretanto, o diâmetro arterial médio da artéria radial foi significativamente diminuído de $2,63 \pm 0,35$ para 2,51 $\pm 0,29 \mathrm{~mm}$ durante o seguimento tardio médio de 4,5 meses $(P=0,01)$, sem diferenças no tempo de acesso vascular e no sucesso do procedimento, quando comparados com a primeira punção, embora com maior incidência de oclusão assintomática da artéria radial $(2,6 \%$ vs. $0 \%$; $P=0,01)$. Em nosso registro, a maioria das reutilizações do acesso radial no caso índice foi precoce em relação ao prévio ou seguinte, muitos deles no dia seguinte a esse procedimento, podendo ter contribuído para a manutenção de alto índice de sucesso e baixa taxa de oclusão assintomática da artéria radial (2,1\%), mesmo com vários procedimentos repetidos em alguns pacientes, além da utilização de introdutores de menor calibre (5 F) na realização da maioria dos procedimentos diagnósticos, que, sabidamente, diminuem a ocorrência de oclusão arterial quando comparados com introdutores de maior calibre. ${ }^{14}$ 

Invasiva. 2010;18(1):37-43.

Em se tratando de um registro observacional, o presente estudo possui como limitações sua natureza não-randômica, o fato de ser conduzido em um centro único e a ausência de seguimento clínico tardio.

\section{CONCLUSÃO}

A reutilização do acesso radial por operadores habituados à técnica na realização de procedimentos coronários diagnósticos e terapêuticos mostra-se efetiva, considerando sua alta taxa de sucesso e a baixa taxa de complicações na maioria dos pacientes, embora com limitação do número de reutilizações a cada procedimento adicional.

\section{CONFLITO DE INTERESSES}

Os autores declararam inexistência de conflito de interesses relacionado a este artigo.

\section{REFERÊNCIAS}

1. Campeau L. Percutaneous radial artery approach for coronary angiography. Cathet Cardiovasc Diagn. 1989;16(1):3-7.

2. Kiemeneij F, Laarman GJ. Percutaneous transradial artery approach for coronary stent implantation. Cathet Cardiovasc Diagn. 1993;30(2):173-8.

3. Louvard Y, Lefevre T, Morice MC. Radial approach: what about the learning curve? Cathet Cardiovasc Diagn. 1997; 42(4):467-8.

4. Labrunie A, Tebet MA, Andrade PB, Andrade MVA, Conterno LO, Mattos LA, et al. Coronariografia via transradial: curva de aprendizagem, avaliada por estudo multicêntrico. Rev Bras Cardiol Invas. 2009;17(1):82-7.

5. Kiemeneij F, Laarman GJ, Odekerken D, Slagboom T, van der Wieken R. A randomized comparison of percutaneous transluminal coronary angioplasty by the radial, brachial and femoral approaches: the access study. J Am Coll Cardiol. 1997;29(6):1269-75
6. Jolly SS, Amlani S, Hamon M, Yusuf S, Mehta SR. Radial versus femoral access for coronary angiography or intervention and the impact on major bleeding and ischemic events: a systematic review and meta-analysis of randomized trials. Am Heart J. 2009;157(1):132-40.

7. Cooper CJ, El-Shiekh RA, Cohen DJ, Blaesing L, Burket MW, Basu A, et al. Effect of transradial access on quality of life and cost of cardiac catheterization: A randomized comparison. Am Heart J. 1999;138(3 Pt 1):430-6.

8. Stella PR, Kiemeneij F, Laarman GJ, Odekerken D, Slagboom $\mathrm{R}$, van der Wieken $\mathrm{R}$. Incidence and outcome of radial artery occlusion following transradial artery coronary angioplasty. Cathet Cardiovasc Diagn. 1997;40:156-8.

9. Bertrand OF, De Larochellière R, Rodés-Cabau J, Proulx G, Gleeton O, Nguyen CM, et al. Early Discharge After Transradial Stenting of Coronary Arteries Study Investigators. A randomized study comparing same-day home discharge and abciximab bolus only to overnight hospitalization and abciximab bolus and infusion after transradial coronary stent implantation. Circulation. 2006;114(24):2636-43. Comment in: Circulation. 2006;114(24):2578-80.

10. Andrade PB, Tebet MA, Andrade MA, Mattos LA, Labrunie A. Segurança e eficácia do acesso radial na realização de procedimentos coronários diagnósticos e terapêuticos em mulheres. Rev Bras Cardiol Invasiva. 2009;17(4):457-62.

11. Caputo RP, Simons A, Giambartolomei A, Grant W, Fedele K, Abraham S, et al. Safety and efficacy of repeat transradial access for cardiac catheterization procedures. Cathet Cardiovasc Intervent. 2001;54:188-90.

12. Sakai H, Ikeda S, Harada T, Yanashiro S, Ozumi K, Ohe H, et al. Limitations of successive transradial approach in the same arm: The Japanese Experience. Cathet Cardiovasc Intervent. 2001;54:204-8.

13. Yoo BS, Lee $S H, K o J Y$, Lee $B K, \operatorname{Kim} S N$, Lee $M O$, et al. Procedural outcomes of repeated transradial coronary procedure. Cathet Cardiovasc Intervent. 2003;58:301-4.

14. Dahm JB, Vogelgesang D, Hummel A, Staudt A, Volzke H, Felix SBA. Randomized trial of 5 vs. 6 French transradial percutaneous coronary interventions. Cathet Cardiovasc Intervent. 2002;57:172-6. 